\title{
Probing FCNC couplings in single top quark production associated with a neutral gauge boson at future lepton colliders
}

\author{
Sara Khatibi讯, Mehrnoosh Moallemi诖 \\ $\dagger$ Department of Physics, University of Tehran, North Karegar Ave., Tehran 14395-547, Iran \\ ${ }^{\ddagger}$ Department of Physics, Isfahan University of Technology, Isfahan 84156-83111, Iran
}

\begin{abstract}
In this paper, we consider a single top quark production in association with a neutral gauge boson ( $\mathrm{Z}$ boson or Photon) at the future electron-positron colliders. We utilize these channels to probe the top quark Flavour Changing Neutral Currents (FCNC) interactions in $t q Z$ and $t q \gamma$ vertices. We perform two separate analyses for top-Z-jet and top- $\gamma$-jet channels. The Standard Model Effective Field Theory (SMEFT) approach is employed to search for these anomalous couplings. We consider parton showering, hadronization and fast detector simulation in our study and use a cut-and-count technique to separate the signal from the Standard Model (SM) background processes. The upper limits on the FCNC couplings at $95 \%$ confidence level for the different integrated luminosities are obtained. It is shown that the future lepton collider would be able to probe the FCNC branching fractions to $\operatorname{Br}(\mathrm{t} \rightarrow \mathrm{q} \gamma)<10^{-4}$ and $\mathrm{Br}(\mathrm{t} \rightarrow \mathrm{qZ})<10^{-4}$ with $3 \mathrm{ab}^{-1}$ of integrated luminosity of data at a center of mass energy of $350 \mathrm{GeV}$.
\end{abstract}

\footnotetext{
${ }^{1}$ sara.khatibi@ut.ac.ir

${ }^{2}$ mehrnoosh.moalemi@gmail.com
} 


\section{Introduction}

The Standard Model (SM) of particle physics has been found to be a successful theory in describing particles and their interactions at $\mathrm{TeV}$ scale. So far, all experimental results are in a good agreement with the SM predictions. However, the SM fails to answer some major experimental observations like the need for the existence of Dark Matter (DM) in the cosmos, the massive neutrinos, baryon asymmetry in the universe, etc. Hence, particle physicists have looked for new physics for several decades.

Despite all the plentiful efforts at the Large Hadron Collider (LHC) at run I and run II, no signal of new physics has been found yet. Therefore, it seems that the new physics scale should be well separated from the electroweak scale and the new degrees of freedom cannot be produced directly at the colliders. So, in order to study the new physics, we should look for indirect signs such as new interactions between the SM particles. Flavour changing neutral current (FCNC) interactions are a good place to seek for these new effects since they are forbidden at the tree level in the SM framework and they are also strongly suppressed at the loop level by the GlashowIliopoulos-Maiani (GIM) mechanism [1].

Particularly, the search for FCNC interactions in the top sector is well-motivated, because of the especial properties of top quark. The SM predicts very small branching ratios $\left(\mathcal{O}\left(10^{-14}\right)\right)$ for different top quark FCNC decay modes [2], hence the current and future experiments would not be able to probe such tiny values. Nevertheless, there are many Beyond Standard Models (BSM) such as Technicolor, Minimal Supersymmetric Standard Model (MSSM), two Higgs Doublet Models (2HDM) that anticipate for large branching ratios for the top FCNC decay modes [3 9]. Therefore, any footprint of the top FCNC interactions can prove the existence of the new physics.

There are several phenomenological papers that studied the top FCNC couplings at different colliders in the literature 10 20. Furthermore, there are many experimental analyses that look for the top FCNC interactions using different channels $21-32$. For instance, the CMS collaboration has studied the top quark FCNC interactions in the anomalous single top quark production in association with a photon in proton-proton collision at $8 \mathrm{TeV}$ 24. Following upper limits at the $95 \% \mathrm{CL}$ on the anomalous top branching ratios have been found; $\operatorname{Br}(\mathrm{t} \rightarrow \mathrm{u} \gamma)<1.3 \times 10^{-4}$ and $\operatorname{Br}(\mathrm{t} \rightarrow \mathrm{c} \gamma)<1.7 \times 10^{-3}$. Recently, more stringent bounds on these branching ratios have been found by ATLAS detector at a center of mass energy of $13 \mathrm{TeV} ; \operatorname{Br}(\mathrm{t} \rightarrow \mathrm{u} \gamma)<6.1 \times 10^{-5}$ and $\operatorname{Br}(\mathrm{t} \rightarrow \mathrm{c} \gamma)<18 \times 10^{-5}[25]$. In addition, the ATLAS experiment has searched for the FCNC couplings in proton-proton collision at $13 \mathrm{TeV}$ by using top-quark pair events which one of them decaying through $t \rightarrow q Z$ and another one decays through the standard mode [26]. The collaboration has found the upper limits at the $95 \%$ CL on the top branching ratios as $\mathrm{Br}(\mathrm{t} \rightarrow \mathrm{uZ})<1.7 \times 10^{-4}$ and $\mathrm{Br}(\mathrm{t} \rightarrow \mathrm{cZ})<2.4 \times 10^{-4}$. Some papers studied the FCNC couplings in the HL-LHC, the reported values for branching ratios are $\operatorname{Br}(\mathrm{t} \rightarrow \mathrm{u} \gamma)<4.6 \times 10^{-5}$, $\operatorname{Br}(\mathrm{t} \rightarrow \mathrm{c} \gamma)<3.4 \times 10^{-4}, \operatorname{Br}(\mathrm{t} \rightarrow \mathrm{uZ})\left(\sigma_{\mu \nu}\right)<8.1 \times 10^{-4}$ and $\operatorname{Br}(\mathrm{t} \rightarrow \mathrm{uZ})\left(\gamma_{\mu}\right)<1.7 \times 10^{-3} 33$ 35].

There are several proposed future electron-positron colliders for very precise measurements such as the future circular electron-positron collider (FCC-ee) [36, 37], the Circular electronpositron Collider (CEPC) [38], the International Linear Collider (ILC) [39 41], and the Compact Linear Collider (CLIC) [42]. Since large amounts of production of top quarks would happen at these colliders, the top quark couplings can be probed at high precision. Our main purpose here is to study the top FCNC couplings in the single top quark production in association with a neutral gauge boson ( $\mathrm{Z}$ or $\gamma$ ) at a future electron-positron collider. This analysis is a complementary channel in addition to other channels to probe the anomalous FCNC couplings, since the top-Z/ $\gamma$ jet has a different signature at the lepton colliders.

In this paper, these signals are studied separately. In the top-Z-jet analysis, we consider both 
top quark and $\mathrm{Z}$ boson decay leptonically, therefore, we expect to have three charged leptons, a b-jet, a light-jet, and the missing energy in the final state. However, in the top- $\gamma$-jet analysis, leptonic decay for the top quark is assumed, so the final state comprises a charged lepton, a hard photon, a b-jet, a light-jet, and the missing energy due to the neutrino. Furthermore, parton showering and hadronization as well as the detector simulations are taken into account in these studies. Then, we determine the upper limits on the top FCNC couplings and the corresponding branching ratios in the both analyses.

The paper is structured as follows. In section 2, we introduce the effective Lagrangian which describes the top quark FCNC interactions with the neutral gauge bosons. In section 3, we explain our analysis strategy like event simulations and event selections for top-Z-jet signal and then present our obtained limits on the new couplings and the corresponding branching ratios. Analysis strategy for top- $\gamma$-jet signal and the related upper limits on the anomalous couplings are described in section 4. Finally, we present our summary in section 5.

\section{Theoretical formalism}

In this section, we explain the theoretical framework which we use to search for FCNC couplings. There are two different approaches to study any new physics at the colliders. The first approach is the direct searches for the well-motivated BSM models. In another word, we look for the new degrees of freedom which anticipated by these new models at the colliders. The second approach is the indirect searches which mean we seek for the new interactions between SM particles.

Since all the experimental measurements are in good agreement with the SM predictions, the new physics scales seem to be well separated from the SM scale. On the other hand, because of the presence of several new physics scenarios and sometimes with the same signatures at the colliders, an economical way to search for the new physics would be using a model independent way. The Standard Model Effective Field Theory (SMEFT) approach provides this model independent way to look for any sign of new physics [43].

In the SMEFT, all the new degrees of freedom are integrated out and the new physics effects show up in the higher dimensional effective operators which contain the new interactions between the SM field content. These higher dimensional operators are suppressed by the inverse powers of the scale of new physics and respect the Lorentz and SM gauge symmetries. The operators with odd dimensions are not considered by assuming baryon and lepton number conservation. Furthermore, the EFT approach is useful when the scale of new physics is much larger than the energy scale of the process. Since the operators are suppressed by the powers of $\Lambda$, one expects that the smallest dimension provides the leading corrections to the SM amplitudes. The effects of dimension eight and higher-order operators are suppressed by at least $1 / \Lambda^{4}$. So, the dimension six operators have dominant contributions to the observables at the colliders. Worth mentioning that, if the coefficients of dimension six operators, which can contribute to the special process, are strongly constrained by current experiments, the dimension eight operators have a main contribution to the process. The SMEFT Lagrangian up to dimension six operators could be written as following [44]:

$$
\mathcal{L}_{\mathrm{SMEFT}}=\mathcal{L}_{\mathrm{SM}}+\sum_{i} \frac{C_{i} O_{i}}{\Lambda^{2}}
$$

here the first term is the SM Lagrangian with four dimensional operators and $O_{i}$ demonstrates operators with dimension six. The new physics scale is denoted by $\Lambda$ and $C_{i}$ are the Wilson couplings. The following dimension six operators can contribute in the FCNC interactions of a 
top quark and a Z boson/photon with an up-type quark (u or c quark) [45]:

$$
\begin{aligned}
O_{u W}^{i j} & =\left(\bar{q}_{L i} \sigma^{\mu \nu} \tau^{I} u_{R j}\right) \tilde{\phi} W_{\mu \nu}^{I}, \\
O_{u B \phi}^{i j} & =\left(\bar{q}_{L i} \sigma^{\mu \nu} u_{R j}\right) \tilde{\phi} B_{\mu \nu}, \\
O_{\phi q}^{(3, i j)} & =i\left(\phi^{\dagger} \tau^{I} D_{\mu} \phi\right)\left(\bar{q}_{L i} \gamma^{\mu} \tau^{I} q_{L j}\right), \\
O_{\phi q}^{(1, i j)} & =i\left(\phi^{\dagger} D_{\mu} \phi\right)\left(\bar{q}_{L i} \gamma^{\mu} q_{L j}\right), \\
O_{\phi u}^{i j} & =i\left(\phi^{\dagger} D_{\mu} \phi\right)\left(\bar{u}_{R i} \gamma^{\mu} u_{R j}\right),
\end{aligned}
$$

where $q_{L i}$ and $u_{R j}$ are left-handed quark doublet and right-handed quark singlet, respectively, and $i, j$ are flavour indices. The Pauli matrices are presented with $\tau^{I}$ and $D_{\mu}$ is covariant derivative. Moreover, $\tilde{\phi}=i \tau^{2} \phi^{*}$ that $\phi$ is the SM Higgs doublet. Their contribution to the FCNC interactions can be parametrized as follows,

$$
\begin{aligned}
\mathcal{L}_{\mathrm{FCNC}}=\sum_{q=u, c}\{ & -\frac{g_{W}}{2 c_{W}} \bar{q} \gamma^{\mu}\left(X_{t q}^{L} P_{L}+X_{t q}^{R} P_{R}\right) t Z_{\mu}+\frac{g_{W}}{4 c_{W} m_{Z}} \bar{q} \sigma^{\mu \nu}\left(\kappa_{t q}^{L} P_{L}+\kappa_{t q}^{R} P_{R}\right) t Z_{\mu \nu} \\
& \left.+\frac{e}{2 m_{t}} \bar{q} \sigma^{\mu \nu}\left(\lambda_{t q}^{L} P_{L}+\lambda_{t q}^{R} P_{R}\right) t A_{\mu \nu}\right\}+ \text { h.c. },
\end{aligned}
$$

where the field strengths of the $\mathrm{Z}$ boson and photon are shown by $Z_{\mu \nu}$ and $A_{\mu \nu}$, respectively. Also, $P_{R}$ and $P_{L}$ demonstrate the right and left-handed projection operators, respectively. The dimensionless anomalous couplings between a top quark, a light up-type quark and a Z boson are presented by $X_{t q}^{L}, X_{t q}^{R}, \kappa_{t q}^{L}$ and $\kappa_{t q}^{R}$. Similarly, $\lambda_{t q}^{L}$ and $\lambda_{t q}^{R}$ are dimensionless anomalous couplings between a top quark, a light up-type quark and a photon. These dimensionless anomalous couplings have the following relation with the Wilson coefficients of effective operators [45],

$$
\begin{aligned}
X_{t q}^{L} & =\frac{1}{2}\left[C_{\phi q}^{(3, j 3)}+C_{\phi q}^{(3,3 j) *}-C_{\phi q}^{(1, j 3)}-C_{\phi q}^{(1,3 j) *}\right] \frac{v^{2}}{\Lambda^{2}} \\
X_{t q}^{R} & =-\frac{1}{2}\left[C_{\phi u}^{j 3}+C_{\phi u}^{3 j *}\right] \frac{v^{2}}{\Lambda^{2}}, \\
\kappa_{t q}^{L} & =\sqrt{2}\left[c_{W} C_{u W}^{3 j *}-s_{W} C_{u B \phi}^{3 j *}\right] \frac{v^{2}}{\Lambda^{2}}, \\
\kappa_{t q}^{R} & =\sqrt{2}\left[c_{W} C_{u W}^{j 3}-s_{W} C_{u B \phi}^{j 3}\right] \frac{v^{2}}{\Lambda^{2}} \\
\lambda_{t q}^{L} & =\frac{\sqrt{2}}{e}\left[s_{W} C_{u W}^{3 j *}+c_{W} C_{u B \phi}^{3 j *}\right] \frac{v m_{t}}{\Lambda^{2}} \\
\lambda_{t q}^{R} & =\frac{\sqrt{2}}{e}\left[s_{W} C_{u W}^{j 3}+c_{W} C_{u B \phi}^{j 3}\right] \frac{v m_{t}}{\Lambda^{2}}
\end{aligned}
$$

where $j$ can be 1 and 2 corresponding to u-quark and c-quark couplings, respectively.

As mentioned before, we are interested to look for the single top production in association with a $\mathrm{Z}$ boson or a photon and a light jet at a future electron-positron collider. This process cannot be produced in the SM framework, however, above FCNC interaction terms allow to generate such processes. Some Feynman diagrams of these processes in presence of the FCNC couplings are illustrated in Fig. 1. Diagrams $a, b$, and $c$ arise from the anomalous couplings $t q Z\left(X_{t q}\right.$ or $\left.\kappa_{t q}\right)$ and diagrams $d, e$, and $f$ occur in presence of the anomalous couplings $t q \gamma\left(\lambda_{t q}\right)$. We should mention that the $e^{+} e^{-} \rightarrow t \bar{t} \rightarrow t V j(V=Z$ or $\gamma)$ process have contribution in our final state and we considered it in our analysis as well (diagrams $c$ and $f$ ). 

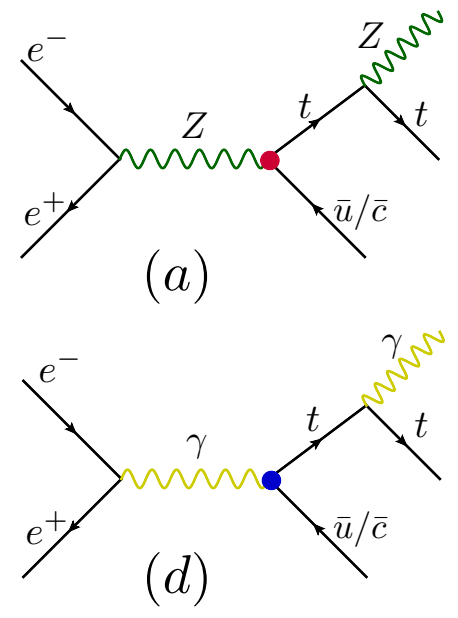

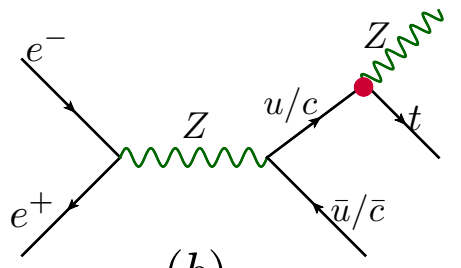

(b)

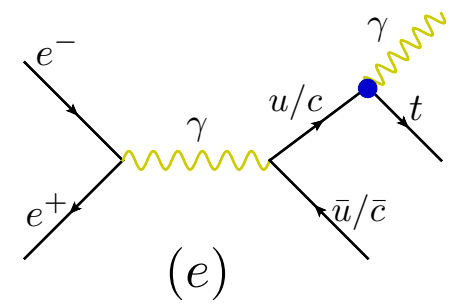

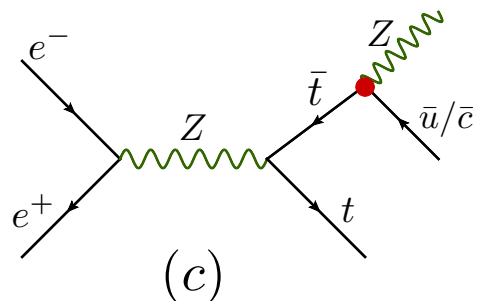

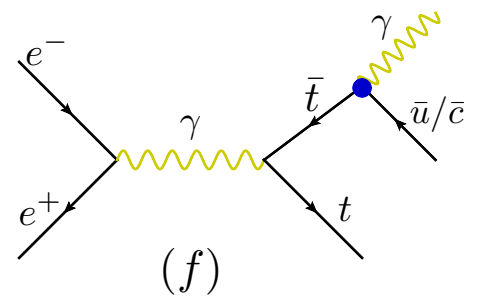

Figure 1: Representative Feynman diagrams for the single top quark production in association with a neutral gauge boson in the presence of the FCNC couplings are shown. Diagrams $a, b$, and $c$ arise from the anomalous couplings $t Z q\left(X_{t q}\right.$ or $\left.\kappa_{t q}\right)$ and diagrams $d, e$, and $f$ occur in presence of the anomalous couplings $t \gamma q\left(\lambda_{t q}\right)$.

In the two next sections, we use the effective Lagrangian (Eq. 3) to generate and analyse events for the top quark production with a neutral gauge boson signal to study FCNC interactions. We explain extensively analysis for the top-Z-jet signal in section 3 and analysis for the top- $\gamma$-jet signal in 4

\section{Analysis Strategy for top-Z-jet signal}

In this section, we study the single top quark production in association with a $\mathrm{Z}$ boson to look for the $t q Z$ and $t q \gamma$ anomalous couplings at the future electron-positron collider. In the first subsection, event generation and simulation for both the signal and backgrounds are explained in detail. Then, we describe our reconstruction method of the final state particles and introduce some strategies to reduce the number of background events with respect to the number of signal events. Eventually, the upper limit which can be extracted from the top-Z-jet signal is presented.

\subsection{Event Generation and Simulation}

Now we are ready to define the top-Z-jet signal and related background processes and describe the event generation and simulation method. Since the mass of final state particles, the top quark and $\mathrm{Z}$ boson, are heavy to be produced in the collider with $\sqrt{s}=240 \mathrm{GeV}$, we just focus on the center of mass energy of $\sqrt{s}=350 \mathrm{GeV}$ in this analysis. The signal process is single top production in association with a $\mathrm{Z}$ boson and a light jet $\left(e^{-} e^{+} \rightarrow t / \bar{t} Z j\right)$, which both top quark and Z boson decay leptonically. As a result, the final state consists of three charged leptons (electron or muon), a b-jet, a light jet, and the missing energy. The representative Feynman diagrams of the signal production are presented in Fig. 1 $(a, b$ and $c$ ). Regarding generate of the signal events, we insert the UFO module [46] in the MADGRAPH 5 package [47]. This UFO module has been obtained from implementing the effective Lagrangian of Eq. 3 [48] in the FEYNRulEs package [49]. 


\begin{tabular}{|c|ccc|cccc|}
\hline Cross-sections (in fb) & \multicolumn{3}{|c|}{ Signal } & \multicolumn{3}{c|}{ Background } \\
\hline Cuts & $t q Z\left(\sigma_{\mu \nu}\right)$ & $t q Z\left(\gamma_{\mu}\right)$ & $t q \gamma$ & $W W j j$ & $W Z j j$ & $t \bar{t}$ & $Z Z j j$ \\
\hline \hline Preselection cuts & $13.5\left(\kappa_{t q}\right)^{2}$ & $9.28\left(X_{t q}\right)^{2}$ & $0.14\left(\lambda_{t q}\right)^{2}$ & 10.12 & 0.26 & 9.25 & 0.007 \\
1 light jet + 1b jet & $6.92\left(\kappa_{t q}\right)^{2}$ & $4.76\left(X_{t q}\right)^{2}$ & $0.066\left(\lambda_{t q}\right)^{2}$ & 4.43 & 0.012 & 4.2 & 0.001 \\
3 leptons & $3.55\left(\kappa_{t q}\right)^{2}$ & $2.5\left(X_{t q}\right)^{2}$ & $0.03\left(\lambda_{t q}\right)^{2}$ & 0.00014 & 0.006 & 0.00024 & 0.0007 \\
$\not p_{T}>30 \mathrm{GeV}$ & $2.6\left(\kappa_{t q}\right)^{2}$ & $1.8\left(X_{t q}\right)^{2}$ & $0.02\left(\lambda_{t q}\right)^{2}$ & 0.00012 & 0.004 & 0.00022 & 0.00001 \\
\hline
\end{tabular}

Table 1: Cross sections for the top-Z-jet signal and the SM background processes before and after passing sequential preliminary cuts at the center of mass energy of $\sqrt{s}=350 \mathrm{GeV}$.

Since all the $t q Z$ and $t q \gamma$ FCNC interactions produce our favorable signal (top-Z-jet), the signal events are categorized into three scenarios. In the first scenario, we choose a non zero value for $\kappa_{t q}^{R}=\kappa_{t q}^{L}=\kappa_{t q}$ and put zero for other anomalous couplings. In the second one, we put zero value for all FCNC couplings except $X_{t q}^{R}=X_{t q}^{L}=X_{t q}$. And in the final case, just $\lambda_{t q}^{R}=\lambda_{t q}^{L}=\lambda_{t q}$ coupling has a non zero value. Also, it should be mentioned that we set the same values for the u-quark and c-quark anomalous couplings.

According to the final state of the signal, the main background processes are $W^{+} W^{-} j j$, $W^{ \pm} Z j j, t \bar{t}$, and $Z Z j j$. In this processes all the W-boson, Z-boson, and top quark decay leptonically. Since tau lepton can have a hadronic decay, it indicates a different signature in the detector, so decay to electron and muon are just taken into account. The MADGRAPH 5 package is used to generate all the background processes. At generator level, preselection cuts like $p_{T} \geq 10 \mathrm{GeV}$ and $|\eta|<3$ are applied on all objects in the final state ${ }^{3}$ The angular distance between all the charged leptons and jets has to be greater than $0.4\left(\Delta R=\sqrt{(\Delta \eta)^{2}+(\Delta \phi)^{2}}\right)$. For both signal and backgrounds, the PYTHIA package [50] is employed for parton showering and hadronization. Then, the Delphes package [51] is utilized to model the detector performance with using the ILD card [40]. For numerical calculation, we use following the SM inputs: $m_{\text {top }}=173.3 \mathrm{GeV}$, $m_{Z}=91.187 \mathrm{GeV}, m_{W}=80.419 \mathrm{GeV}, \alpha=1 / 127.90$, and $s_{W}^{2}=0.234[52$.

In order to select the signal events, we desire to have at least two jets with $p_{T} \geq 20 \mathrm{GeV}$ and $|\eta|<2.4$, which at least one of them is required to be tagged as a b-jet. We require the events with at least three charged leptons with $p_{T} \geq 10 \mathrm{GeV}$ and $|\eta|<2.4$. Furthermore, we choose events with the missing transverse momentum greater than $30 \mathrm{GeV}$. The cross sections including the branching ratios for three signal scenarios and the corresponding SM backgrounds after passing sequential preliminary cuts at the center of mass energy of $350 \mathrm{GeV}$ are presented in Table 1 . It is worth mentioning that the reported cross sections for signal and background processes are not included the QED ISR. Moreover, it's noteworthy that the gamma-induced processes or egamma processes can be the new sources of background, however, we expect that by applying our preliminary and secondary cuts their contribution will be strongly suppressed. So, we don't consider them in our analysis.

As it is clear, the cross section of the signal for $t q \gamma$ scenario is significantly less than two others, so we expect that the extracting upper limit for this coupling should be looser than two others. Also, the cross section of the signal for $t q Z\left(\sigma_{\mu \nu}\right)$ scenario is greater than $t q Z\left(\gamma_{\mu}\right)$, because of the presence of the gauge boson's momentum in the $t q Z\left(\sigma_{\mu \nu}\right)$ interaction term. The preliminary cuts efficiencies for $t q Z\left(\sigma_{\mu \nu}\right), t q Z\left(\gamma_{\mu}\right)$, and $t q \gamma$ signal scenarios are $0.19,0.19$, and 0.14 , respectively.

It is notable that, although the production cross sections for some background processes like $W W j j$ and $t \bar{t}$ are large at parton level, they are remarkably reduced after applying preliminary

\footnotetext{
${ }^{3}$ For applying cuts we use $p_{T}$ and $\eta$, however, we should mention that at sub-TeV lepton colliders, energy and angle are much more standard variables.
} 

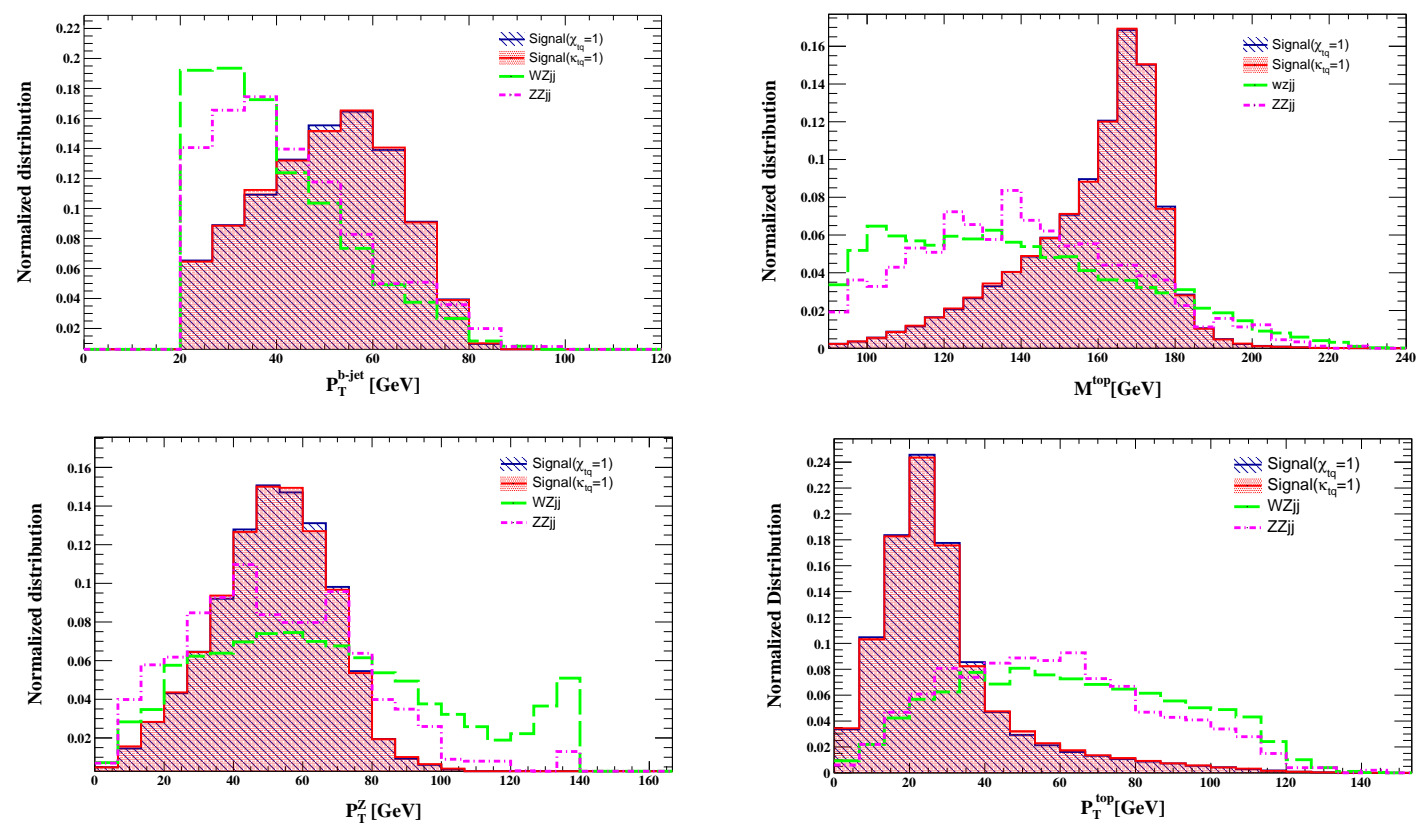

Figure 2: The kinematic distributions of the top-Z-jet signal (for $t q Z\left(\sigma_{\mu \nu}\right)$ and $t q Z\left(\gamma_{\mu}\right)$ scenarios) and the main backgrounds, $W Z j j$ and $Z Z j j$, at the center of mass energy of $\sqrt{s}=350 \mathrm{GeV}$.

cuts, particularly, when we require exactly three charged leptons at the final state. The preliminary cuts efficiencies for $W W j j, W Z j j, t \bar{t}$, and $Z Z j j$ backgrounds are at the order of $10^{-5}, 10^{-2}, 10^{-4}$, and $10^{-3}$, respectively. Therefore, our main backgrounds are $W Z j j$ and $Z Z j j$ channels in this analysis.

\subsection{Separation of Signal from Background}

For suppressing the contributions of background events, we look at different kinematic distributions to find suitable secondary cuts. Therefore, we need to reconstruct the top quark and Z boson of the final state. In order to reconstruct the $\mathrm{Z}$ boson, we require two opposite charged leptons with an invariant mass between 60 to $120 \mathrm{GeV}$. Then the top quark will be reconstructed with the remaining charged lepton, the b-jet and the neutrino. As it is clear from Table 1 that the signal cross sections for $\kappa_{t q}$ and $X_{t q}$ couplings are larger than the signal cross section for $\lambda_{t q}$, so we first describe our secondary cuts for $t q Z$ interactions $\left(\sigma_{\mu \nu}\right.$ and $\left.\gamma_{\mu}\right)$.

Fig. 2 illustrates some kinematic distributions of the top-Z-jet signal and the main background processes, $W Z j j$ and $Z Z j j$, after preliminary cuts and top quark and $\mathrm{Z}$ boson reconstruction. Figures upper right and lower right show the mass and transverse momentum of the reconstructed top quark, respectively. Figures upper left and lower left illustrate the transverse momentum of the $\mathrm{b}$-jet and the reconstructed $\mathrm{Z}$ boson, respectively.

To separate more background from signal events, we apply further cuts according to the distributions in Fig. 2. From the top quark's mass distribution (upper right), it is clear that the signal has a prominent peak around the nominal top mass. A window cut on the reconstructed top quark mass $\left(140<\mathrm{M}^{\text {top }}<180 \mathrm{GeV}\right)$ causes to suppress backgrounds without a real top quark in the final state. According to the top quark's transverse momentum distribution (lower right), the signal's peak is around $20 \mathrm{GeV}$, however, the main backgrounds have more spread distributions 


\begin{tabular}{|c|c|c|c|c|}
\hline Cross-sections (in fb) & \multicolumn{2}{|c|}{ Signal } & \multicolumn{2}{|c|}{ Background } \\
\hline Cuts & $t q Z\left(\sigma_{\mu \nu}\right)$ & $t q Z\left(\gamma_{\mu}\right)$ & $W Z j j$ & $Z Z j j$ \\
\hline \hline$p_{T}^{\text {top }}<30 \mathrm{GeV}$ & $2.17\left(\kappa_{t q}\right)^{2}$ & $1.54\left(X_{t q}\right)^{2}$ & 0.001 & $4.2 \times 10^{-6}$ \\
$p_{T}^{Z}<80 \mathrm{GeV}$ & $2.14\left(\kappa_{t q}\right)^{2}$ & $\left.1.52 X_{t q}\right)^{2}$ & 0.0009 & $4 \times 10^{-6}$ \\
$p_{T}^{\text {b-jet }}>30 \mathrm{GeV}$ & $1.9\left(\kappa_{t q}\right)^{2}$ & $1.37\left(X_{t q}\right)^{2}$ & 0.0007 & $3.1 \times 10^{-6}$ \\
$140<\mathrm{M}^{\text {top }}<180 \mathrm{GeV}$ & $1.7\left(\kappa_{t q}\right)^{2}$ & $1.14\left(X_{t q}\right)^{2}$ & 0.0003 & $1.7 \times 10^{-6}$ \\
\hline
\end{tabular}

Table 2: Cross sections for the top-Z-jet signal (for $t q Z\left(\sigma_{\mu \nu}\right)$ and $t q Z\left(\gamma_{\mu}\right)$ scenarios) and the SM main background processes $(W Z j j$ and $Z Z j j)$ after passing sequential secondary cuts at the center of mass energy of $\sqrt{s}=350 \mathrm{GeV}$.

which extend up to around $120 \mathrm{GeV}$. Therefore, the transverse momentum of the top quark is required not to be greater than $30 \mathrm{GeV}$. Moreover, according to the b-jet's transverse momentum distribution (upper left), we accept events with $p_{T}^{\mathrm{b}-\text { jet }}>30 \mathrm{GeV}$. Also, we apply a cut on the $\mathrm{Z}$ boson's transverse momentum distribution (lower left) to alleviate the number of background events which is required not to be greater than $80 \mathrm{GeV}$.

The cross sections for the signal scenarios and the corresponding SM backgrounds after passing sequential secondary cuts at $\sqrt{s}=350 \mathrm{GeV}$ are shown in Table 2. The secondary cuts efficiencies for $t q Z\left(\sigma_{\mu \nu}\right)$ and $t q Z\left(\gamma_{\mu}\right)$ signal scenarios are 0.13 and 0.12 while those for $W Z j j$ and $Z Z j j$ backgrounds are 0.001 and 0.0002 , respectively.

The same method is used to find the secondary cuts and final efficiencies for $t q \gamma$ signal scenario. Form the same kinematic distribution as before, we extract following cuts: $p_{T}^{\text {top }}<80 \mathrm{GeV}$, $p_{T}^{Z}<80 \mathrm{GeV}, p_{T}^{\mathrm{b}-\text { jet }}>30 \mathrm{GeV}$, and $140<\mathrm{M}^{\text {top }}<180 \mathrm{GeV}$.Then, we find the corresponding efficiencies as; $\epsilon_{t q \gamma}=0.084, \epsilon_{W Z j j}=0.002$, and $\epsilon_{Z Z j j}=0.0004$.

\subsection{Sensitivity Estimation}

In this subsection, we present the upper limits on the FCNC couplings ( $t q Z$ and $t q \gamma)$ at a confidence level (CL) of $95 \%$ by analyzing the single top quark production in association with a $\mathrm{Z}$ boson. To estimate the sensitivity of the future electron-positron collider to these anomalous couplings, we employ a Bayesian approach using a flat prior distribution [52,53. A Poisson distribution is assumed for the probability of observing $n_{\text {obs }}$ events,

$$
L\left(n_{\mathrm{obs}}, n_{\mathrm{S}}, n_{\mathrm{B}}\right)=\frac{\left(n_{\mathrm{S}}+n_{\mathrm{B}}\right)^{n_{\mathrm{obs}}}}{n_{\mathrm{obs}} !} e^{-\left(n_{\mathrm{S}}+n_{\mathrm{B}}\right)} .
$$

Notice that in the above equation, $n_{\mathrm{S}}$ is defined by $n_{\mathrm{S}}=\epsilon_{\mathrm{S}} \times \mathcal{L} \times \sigma_{\mathrm{S}}$, that $\epsilon_{\mathrm{S}}$ is the efficiency of the signal after all cuts and $\mathcal{L}$ is the integrated luminosity. For a given integrated luminosity and backgrounds cross section (Table 1, first row), the number of expected background events are given by $n_{\mathrm{B}}=\epsilon_{\mathrm{B}} \times \mathcal{L} \times \sigma_{\mathrm{B}}$, which $\epsilon_{\mathrm{B}}$ is the efficiency of the background processes after all the preliminary and secondary cuts. The upper limit on the number of signal process of $e^{-} e^{+} \rightarrow t Z j$ can be obtained from the following equation at 95\% CL:

$$
\frac{95}{100}=\frac{\int_{0}^{n_{\text {limit }}} L\left(n_{\mathrm{obs}}, n_{\mathrm{S}}, n_{\mathrm{B}}\right) \mathrm{d} n_{\mathrm{S}}}{\int_{0}^{\infty} L\left(n_{\mathrm{obs}}, n_{\mathrm{S}}, n_{\mathrm{B}}\right) \mathrm{d} n_{\mathrm{S}}}
$$

We assume that the observed number of events at the detectors are consistent with the expected number of background events and solve above equation to find the upper limit on the number of signal events. This limit can be translated to the upper limit on the signal cross section and 


\begin{tabular}{|c||c||c|}
\hline Integrated luminosity & Coupling & Branching ratio \\
\hline \hline \multirow{3}{*}{$300 \mathrm{fb}^{-1}$} & $\kappa_{t q}<0.07$ & $\operatorname{Br}(\mathrm{t} \rightarrow \mathrm{qZ})\left(\sigma_{\mu \nu}\right)<1.8 \times 10^{-3}$ \\
& $X_{t q}<0.09$ & $\operatorname{Br}(\mathrm{t} \rightarrow \mathrm{qZ})\left(\gamma_{\mu}\right)<3.8 \times 10^{-3}$ \\
& $\lambda_{t q}<0.91$ & $\operatorname{Br}(\mathrm{t} \rightarrow \mathrm{q} \gamma)<3.5 \times 10^{-1}$ \\
\hline \hline & $\kappa_{t q}<0.04$ & $\operatorname{Br}(\mathrm{t} \rightarrow \mathrm{qZ})\left(\sigma_{\mu \nu}\right)<5.8 \times 10^{-4}$ \\
& $X_{t q}<0.05$ & $\operatorname{Br}(\mathrm{t} \rightarrow \mathrm{qZ})\left(\gamma_{\mu}\right)<1.2 \times 10^{-3}$ \\
& $\lambda_{t q}<0.50$ & $\operatorname{Br}(\mathrm{t} \rightarrow \mathrm{q} \gamma)<1.1 \times 10^{-1}$ \\
\hline & $\kappa_{t q}<0.028$ & $\operatorname{Br}(\mathrm{t} \rightarrow \mathrm{qZ})\left(\sigma_{\mu \nu}\right)<2.8 \times 10^{-4}$ \\
& $X_{t q}<0.03$ & $\operatorname{Br}(\mathrm{t} \rightarrow \mathrm{qZ})\left(\gamma_{\mu}\right)<4.2 \times 10^{-4}$ \\
& $\lambda_{t q}<0.33$ & $\operatorname{Br}(\mathrm{t} \rightarrow \mathrm{q} \gamma)<4.6 \times 10^{-2}$ \\
\hline
\end{tabular}

Table 3: The upper limits and the corresponding branching ratios at 95\% CL on the $t q Z\left(\sigma_{\mu \nu}\right.$ and $\gamma_{\mu}$ ) and $t q \gamma$ at the center of mass energies $\sqrt{s}=350 \mathrm{GeV}$ for different integrated luminosity of $300 \mathrm{fb}^{-1}, 1 \mathrm{ab}^{-1}$ and $3 \mathrm{ab}^{-1}$ by studying the top-Z-jet signal.

then to the upper limits on the FCNC couplings. Table 3 presents the upper limits at 95\% CL on the $t q Z\left(\sigma_{\mu \nu}\right.$ and $\left.\gamma_{\mu}\right)$ and $t q \gamma$ couplings at the center of mass energies $350 \mathrm{GeV}$ for different integrated luminosity of $300 \mathrm{fb}^{-1}, 1 \mathrm{ab}^{-1}$ and $3 \mathrm{ab}^{-1}$ by studying the top-Z-jet channel. As we expected before, the upper limit on the $\lambda_{t q}$ is looser than other anomalous couplings. Then, these limits are translated to the corresponding branching which are shown in the table 3 for different integrated luminosities of data. It is worth mentioning that these upper limits on the anomalous coupling can be translated to the bounds on $\Lambda / \sqrt{C_{i}}$ by using Eq. 4 . According to our upper limits on the anomalous coupling, we can roughly say $\Lambda / \sqrt{C_{i}}$ should be greater than $\sim 1.5 \mathrm{TeV}$.

\section{Analysis Strategy for top- $\gamma$-jet signal}

In this section, we analyze the single top quark production in association with a photon process to search for the $t q \gamma$ and $t q Z$ FCNC couplings at the future lepton collider. First of all, we describe event generation and simulation for the signal and background processes. Then, we look at some kinematic distribution to separate the background events from the signal events. Finally, the upper limits which can be obtained from the top- $\gamma$-jet signal are given.

\subsection{Event Generation and Simulation}

Here, we define the top- $\gamma$-jet signal and related SM background processes and describe the event generation and simulation method. In this analysis, we focus on the electron-positron collisions at the center of mass energy of $\sqrt{s}=350 \mathrm{GeV}$. The signal is defined as a single top production in association with a photon and a light jet $\left(e^{-} e^{+} \rightarrow t / \bar{t} \gamma j\right)$, that top quark decays leptonically. Consequently, the final state includes a charged leptons (electron or muon), a hard photon, a b-jet, a light jet, and the missing energy due to the neutrino. Since the photon in the final state is a hard photon, we can handle it to suppress background contributions. Some related Feynman diagrams for top- $\gamma$-jet signal are demonstrated in Fig. 1 ( $(d, e$ and $f)$. Since our desirable signal (top- $\gamma$-jet) is raised up by all the $t q Z$ and $t q \gamma$ FCNC interactions, again we employ three different scenarios to generate signal events; 1) $\left.\lambda_{t q}^{R}=\lambda_{t q}^{L}=\lambda_{t q} \neq 0,2\right) \kappa_{t q}^{R}=\kappa_{t q}^{L}=\kappa_{t q} \neq 0$, and 3) $X_{t q}^{R}=X_{t q}^{L}=X_{t q} \neq 0$.

According to the final state, we consider the following background processes; $W^{ \pm} j j \gamma, Z j j \gamma$, and $t \bar{t} \gamma$. The W-boson and Z-boson decay leptonically in all the above backgrounds, however, for $t \bar{t} \gamma$ semi-leptonic decay is assumed. In all cases, just decay to electron and muon are taken into 


\begin{tabular}{|c|ccc|ccc|}
\hline Cross-sections (in fb) & \multicolumn{3}{|c|}{ Signal } & \multicolumn{3}{c|}{ Background } \\
\hline Cuts & $t q \gamma$ & $t q Z\left(\sigma_{\mu \nu}\right)$ & $t q Z\left(\gamma_{\mu}\right)$ & $W j j \gamma$ & $Z j j \gamma$ & $t \bar{t} \gamma$ \\
\hline \hline Preselection cuts & $182\left(\lambda_{t q}\right)^{2}$ & $230.6\left(\kappa_{t q}\right)^{2}$ & $29.3\left(X_{t q}\right)^{2}$ & 84 & 2.9 & 0.0057 \\
1 light jet + 1b jet & $98.16\left(\lambda_{t q}\right)^{2}$ & $125.9\left(\kappa_{t q}\right)^{2}$ & $15.57\left(X_{t q}\right)^{2}$ & 4.9 & 0.46 & 0.0045 \\
1 Photon & $47.83\left(\lambda_{t q}\right)^{2}$ & $33.3\left(\kappa_{t q}\right)^{2}$ & $4.5\left(X_{t q}\right)^{2}$ & 1.54 & 0.15 & $3 \times 10^{-5}$ \\
1 lepton & $38.26\left(\lambda_{t q}\right)^{2}$ & $26.34\left(\kappa_{t q}\right)^{2}$ & $3.6\left(X_{t q}\right)^{2}$ & 1.2 & 0.06 & $3 \times 10^{-6}$ \\
$\not \not_{T}>30 \mathrm{GeV}$ & $28.4\left(\lambda_{t q}\right)^{2}$ & $19.5\left(\kappa_{t q}\right)^{2}$ & $\left.2.6 X_{t q}\right)^{2}$ & 0.8 & 0.002 & $2 \times 10^{-6}$ \\
\hline
\end{tabular}

Table 4: Cross sections for the top- $\gamma$-jet signal and the SM background processes before and after passing sequential preliminary cuts at center of mass energy of $\sqrt{s}=350 \mathrm{GeV}$.

account. To generate and simulate the signal and background events, we follow the procedure which explained in subsection 3.1.

In order to select the events, at least two jets with $p_{T} \geq 20 \mathrm{GeV}$ and $|\eta|<2.4$ are required, which at least one of them should be tagged as a b-jet. Events including at least one isolated photon with $p_{T} \geq 30 \mathrm{GeV}$ and $|\eta|<2.4$ are selected. Furthermore, we require the events with exactly one charged leptons with $p_{T} \geq 10 \mathrm{GeV}$ and $|\eta|<2.4$. Also, we choose events with missing transverse momentum greater than $30 \mathrm{GeV}$.

The cross sections including the branching ratios for three signal scenarios and the corresponding SM backgrounds after passing sequential preliminary cuts at $\sqrt{s}=350 \mathrm{GeV}$ are presented in Table 4. The preliminary cuts efficiencies for $t q \gamma, t q Z\left(\sigma_{\mu \nu}\right)$, and $t q Z\left(\gamma_{\mu}\right)$ signal scenarios are 0.15 , 0.08 , and 0.09 , respectively. The primary cuts efficiencies for $W j j \gamma, Z j j \gamma$, and $t \bar{t} \gamma$ backgrounds are at the order of $10^{-2}, 10^{-4}$, and $10^{-4}$, respectively. It is notable that these cuts can reduce significantly the number of background events.

It is worth mentioning that there is another source for background for top- $\gamma$-jet channel. Processes like $W^{ \pm} j j, Z j j$, and $t \bar{t}$ which don't have any real photon can mimic the signal since their jets would be misidentified as photons in the detector. The production cross sections for these background processes are large with respect to the other considered backgrounds, however, after above requirements, their efficiencies remarkably reduce. As a result, here we neglect this source of background nevertheless in a more realistic detector simulation their contribution should be taken into account.

\subsection{Separation of Signal over Background}

In order to alleviate the number of the main SM background events, we follow the same way as described in subsection 3.2. Therefore, we focus on some kinematic distributions to find the suitable secondary cuts. Again at the first step, we need to reconstruct the top quark of the final state. Since we have a clean final state, it is straightforward to reconstruct the top quark. The top quark will be reconstructed by combining the charged lepton, the high energetic b-jet and the neutrino. We show the kinematic distributions and describe our obtained secondary cuts and limits for $\lambda_{t q}$ anomalous coupling. Then, we will follow the same procedure to find the upper limits on the $\kappa_{t q}$ and $X_{t q}$ FCNC couplings.

Fig. 3 demonstrates different kinematic distributions of the top- $\gamma$-jet signal and some SM background processes $(W j j \gamma$ and $Z j j \gamma)$ after preselection cuts and top quark reconstruction. Figures upper right and lower right demonstrate mass and the transverse momentum of the reconstructed top quark, respectively. Figures upper left and lower left illustrate the transverse momentum of the light-jet and the photon, respectively.

For suppressing more background events, we apply further cuts according to distributions in 

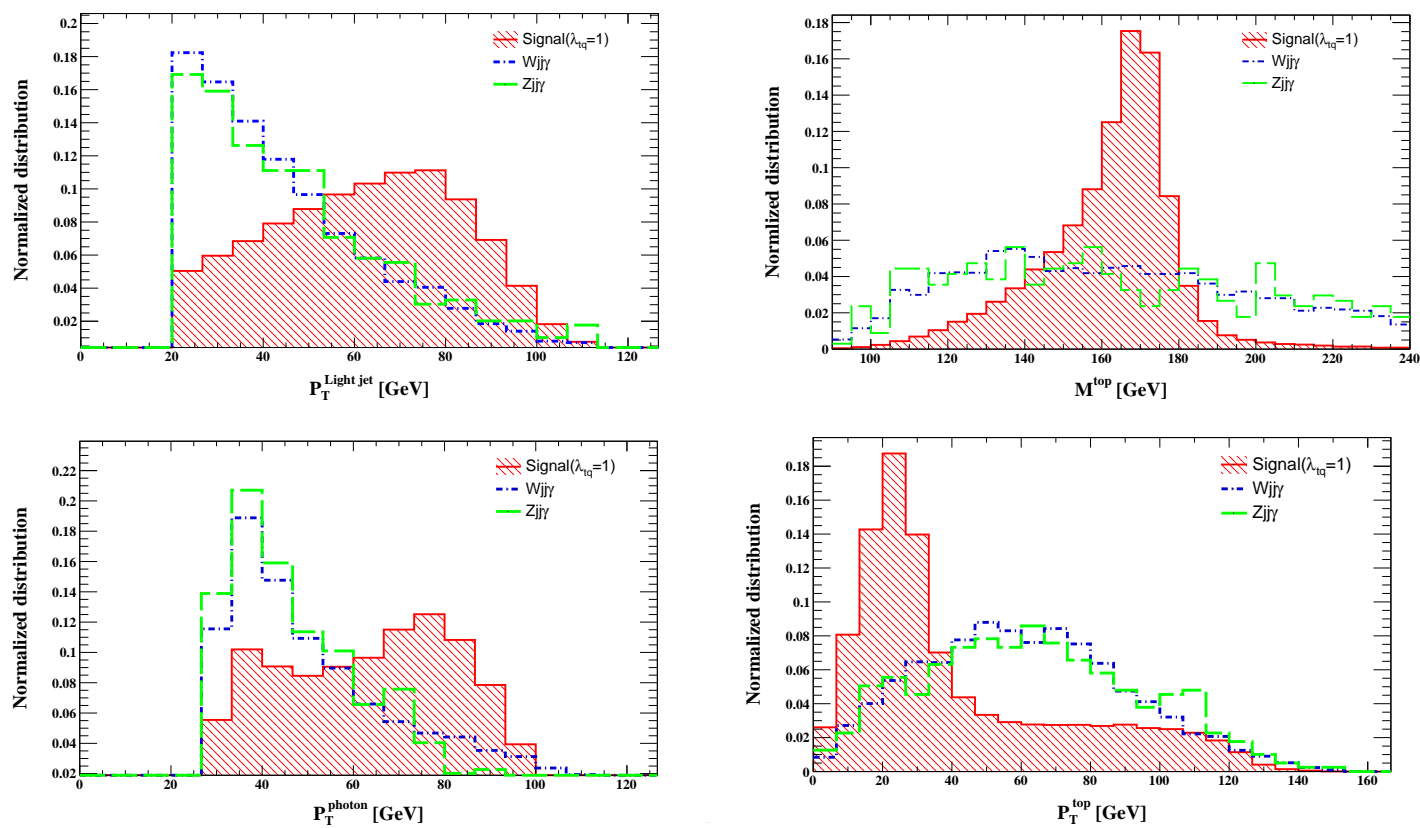

Figure 3: The kinematic distributions of the top- $\gamma$-jet signal (for $t q \gamma$ scenario) and the main backgrounds; $W j j \gamma$ and $Z j j \gamma$ at the center of mass energy of $\sqrt{s}=350 \mathrm{GeV}$.

\begin{tabular}{|c|c|ccc|}
\hline Cross-sections (in fb) & Signal & \multicolumn{3}{|c|}{ Background } \\
\hline Cuts & $t q \gamma$ & $W j j \gamma$ & $Z j j \gamma$ & $t \bar{t} \gamma$ \\
\hline \hline$p_{T}^{\text {top }}<40 \mathrm{GeV}$ & $18.4\left(\lambda_{t q}\right)^{2}$ & 0.2 & $5.7 \times 10^{-4}$ & $1 \times 10^{-6}$ \\
$p_{T}^{\gamma}>40 \mathrm{GeV}$ & $17\left(\lambda_{t q}\right)^{2}$ & 0.13 & $3.3 \times 10^{-4}$ & $4.6 \times 10^{-7}$ \\
$p_{T}^{\text {light-jet }}>40 \mathrm{GeV}$ & $15\left(\lambda_{t q}\right)^{2}$ & 0.07 & $1.8 \times 10^{-4}$ & $2.6 \times 10^{-7}$ \\
$140<\mathrm{M}^{\text {top }}<180 \mathrm{GeV}$ & $12.6\left(\lambda_{t q}\right)^{2}$ & 0.02 & $2.3 \times 10^{-5}$ & $2 \times 10^{-7}$ \\
\hline
\end{tabular}

Table 5: Cross sections for the top- $\gamma$-jet signal (for $t q \gamma$ scenario) and the SM background processes after passing sequential secondary cuts at the center of mass energy of $\sqrt{s}=350 \mathrm{GeV}$.

Fig. 3. The top quark mass distribution (upper right) for the signal shows a clear peak around $170 \mathrm{GeV}$. Therefore, we applied a window cut on the reconstructed top quark mass $\left(140<\mathrm{M}^{\text {top }}<\right.$ $180 \mathrm{GeV}$ ). From the top quark transverse momentum distribution (lower right), the signal's peak is around $20 \mathrm{GeV}$, however, the main backgrounds have more spread distributions which extended up to around $160 \mathrm{GeV}$. So, the transverse momentum of the top quark is required not to be greater than $40 \mathrm{GeV}$.

Furthermore, according to the light-jet's transverse momentum distribution (upper left), we desire events with $p_{T}^{\text {light-jet }}>40 \mathrm{GeV}$. Also, we apply a cut on the hard photon transverse momentum distribution (lower left) to reduce more number of background events which is required to be greater than $40 \mathrm{GeV}$. In addition, according to the jet and b-jet multiplicities, we choose events with number of jets and b-jet less than 3 and 2 , respectively. This requirement can suppress a large number of background events with a high number of jets.

The cross sections for the $t q \gamma$ signal scenarios and the corresponding SM backgrounds after passing sequential secondary cuts are shown in Table 5. The final efficiencies for the $t q \gamma$ signal scenario is 0.07 while those for $W j j \gamma, Z j j \gamma$, and $t \bar{t} \gamma$ backgrounds are at the order of $10^{-4}, 10^{-6}$, 


\begin{tabular}{|c||c||c|}
\hline Integrated luminosity & Coupling & Branching ratio \\
\hline \hline & $\kappa_{t q}<0.065$ & $\operatorname{Br}(\mathrm{t} \rightarrow \mathrm{qZ})\left(\sigma_{\mu \nu}\right)<1.5 \times 10^{-3}$ \\
$300 \mathrm{fb}^{-1}$ & $X_{t q}<0.15$ & $\operatorname{Br}(\mathrm{t} \rightarrow \mathrm{qZ})\left(\gamma_{\mu}\right)<1.1 \times 10^{-2}$ \\
& $\lambda_{t q}<0.04$ & $\operatorname{Br}(\mathrm{t} \rightarrow \mathrm{q} \gamma)<6.8 \times 10^{-4}$ \\
\hline \hline & $\kappa_{t q}<0.047$ & $\operatorname{Br}(\mathrm{t} \rightarrow \mathrm{qZ})\left(\sigma_{\mu \nu}\right)<8 \times 10^{-4}$ \\
& $X_{t q}<0.11$ & $\operatorname{Br}(\mathrm{t} \rightarrow \mathrm{qZ})\left(\gamma_{\mu}\right)<5.7 \times 10^{-3}$ \\
& $\lambda_{t q}<0.03$ & $\operatorname{Br}(\mathrm{t} \rightarrow \mathrm{q} \gamma)<3.87 \times 10^{-4}$ \\
\hline & $\kappa_{t q}<0.035$ & $\operatorname{Br}(\mathrm{t} \rightarrow \mathrm{qZ})\left(\sigma_{\mu \nu}\right)<4.5 \times 10^{-4}$ \\
& $X_{t q}<0.085$ & $\operatorname{Br}(\mathrm{t} \rightarrow \mathrm{qZ})\left(\gamma_{\mu}\right)<3.4 \times 10^{-3}$ \\
& $\lambda_{t q}<0.02$ & $\operatorname{Br}(\mathrm{t} \rightarrow \mathrm{q} \gamma)<1.7 \times 10^{-4}$ \\
\hline
\end{tabular}

Table 6: The upper limits and the corresponding branching ratios at 95\% CL on the $t q Z\left(\sigma_{\mu \nu}\right.$ and $\gamma_{\mu}$ ) and $t q \gamma$ at the center of mass energies $\sqrt{s}=350 \mathrm{GeV}$ for different integrated luminosity of $300 \mathrm{fb}^{-1}, 1 \mathrm{ab}^{-1}$ and $3 \mathrm{ab}^{-1}$ by studying the top- $\gamma$-jet signal.

and $10^{-5}$, respectively. The same method is used to find the secondary cuts and final efficiencies for $t q Z\left(\sigma_{\mu \nu}\right)$ and $t q Z\left(\gamma_{\mu}\right)$ signal scenarios. In the following, these final efficiencies are used to estimate the upper limits on the anomalous couplings.

\subsection{Sensitivity Estimation}

In this part, the obtained upper limits on the FCNC couplings ( $t q Z$ and $t q \gamma)$ at 95\% CL by analyzing the single top quark production in association with photon are presented. Again, we follow the same procedure which explained in subsection 3.3 .

Table 6 shows the upper limits and the corresponding branching ratios at $95 \%$ CL on the $t q Z$ $\left(\sigma_{\mu \nu}\right.$ and $\left.\gamma_{\mu}\right)$ and $t q \gamma$ at the center of mass energy of $350 \mathrm{GeV}$ for different integrated luminosities of $300 \mathrm{fb}^{-1}, 1 \mathrm{ab}^{-1}$ and $3 \mathrm{ab}^{-1}$ using top- $\gamma$-jet signal. Also, we do the same study on top- $\gamma$-jet channel at the center of mass energy of $240 \mathrm{GeV}$ for the integrated luminosity of $10 \mathrm{ab}^{-1}$ and find upper limits at 95\% CL on the branching ratio as follows; $\operatorname{Br}(\mathrm{t} \rightarrow \mathrm{q} \gamma)<2.2 \times 10^{-2}$. It is worth mentioning that the cross section of $t \bar{t}$ at the threshold is too small by a factor of three. So we consider the threshold resummation effect on the $t \bar{t}$ cross section, however, these effects change our result by less than $5 \%$.

\section{Summary}

Flavour Changing Neutral Current (FCNC) interactions in the top quark sector are highly suppressed in the Standard Model (SM) framework. The predicted branching ratios for different top FCNC decay modes are of $\mathcal{O}\left(10^{-14}\right)$ in the SM, while there are some Beyond SM models that anticipate large values for these branching ratios. Therefore, any sign of top quark FCNC interaction would be a promising indication of the existence of new physics. On the other hand, future electron-positron colliders with high luminosity are able to produce large numbers of top quark and give us this opportunity to probe top quark properties with high precision.

In this paper, we study the single top quark production in association with a neutral gauge boson (Z boson or photon) to search for $t q Z$ and $t q \gamma$ anomalous couplings at the future electronpositron collider. Since many BSMs predict the FCNC interactions with the same signatures at particle colliders in many cases, using the model independent approach is more useful. So, we employ the Standard Model Effective Field Theory (SMEFT) approach in this study. We perform two separate analyses for top-Z-jet and top- $\gamma$-jet channels. 
In the top-Z-jet study, we consider leptonic decay of top quark and Z boson. As a result, we have three charged leptons (electron or muon), a light-jet, a b-jet and missing energy in the final state. Since all the FCNC couplings $t q Z\left(\sigma_{\mu \nu}\right), t q Z\left(\gamma_{\mu}\right)$ and $t q \gamma$ can produce our favourable signal, we categorize our signal in three scenarios. We consider all the main SM background processes for this channel. Also, the parton showering and hadronization are taken into account as well as fast detector simulation. Then, we use a cut-and-count technique to separate the signal from the background events. The upper limits at 95\% CL are obtained on the anomalous couplings and the corresponding branching ratios for different the integrated luminosity at the center of mass energy of $350 \mathrm{GeV}$. For example, it is found at the $95 \%$ CL with $3 \mathrm{ab}^{-1}$ luminosity of data; $\operatorname{Br}(\mathrm{t} \rightarrow \mathrm{qZ})\left(\sigma_{\mu \nu}\right)<2.8 \times 10^{-4}, \operatorname{Br}(\mathrm{t} \rightarrow \mathrm{qZ})\left(\gamma_{\mu}\right)<4.2 \times 10^{-4}$, and $\operatorname{Br}(\mathrm{t} \rightarrow \mathrm{q} \gamma)<4.6 \times 10^{-2}$.

In the top- $\gamma$-jet study, leptonically decay of top quark is assumed. So, the final state consists of one charged leptons (electron or muon), a hard photon, a light-jet, a b-jet and the missing energy. The upper limits at $95 \% \mathrm{CL}$ are obtained for $3 \mathrm{ab}^{-1}$ luminosity of data at the center of mass energy $350 \mathrm{GeV}$ are as follows; $\mathrm{Br}(\mathrm{t} \rightarrow \mathrm{qZ})\left(\sigma_{\mu \nu}\right)<4.5 \times 10^{-4}, \mathrm{Br}(\mathrm{t} \rightarrow \mathrm{qZ})\left(\gamma_{\mu}\right)<3.4 \times 10^{-3}$, and $\operatorname{Br}(\mathrm{t} \rightarrow \mathrm{q} \gamma)<1.7 \times 10^{-4}$. Also, it is worth mentioning here that by using polarized beams the obtained upper limits can be improved [54]. Our obtained results would be improved significantly by using the statistical techniques for separating more signal from background events such as multivariate analysis. The combination of the obtained upper limits from the top quark production associated with a neutral gauge boson with other channels also can lead to stringent bounds on the FCNC couplings $(t q Z$ ana $t q \gamma)$, particularly tighter limits would be expected from combination with limits coming from the top+jet final state.

\section{Acknowledgments}

Authors would like to especially thank Mojtaba Mohammadi Najafabadi and Hamzeh Khanpour for many useful discussions and their invaluable comments on the draft. Authors are also thankful

School of Particles and Accelerators, Institute for Research in Fundamental Sciences (IPM) for hospitality.

\section{References}

[1] S. L. Glashow, J. Iliopoulos, and L. Maiani, Weak Interactions with Lepton-Hadron Symmetry, Phys. Rev. D2 (1970) 1285-1292.

[2] Top Quark Working Group Collaboration, K. Agashe et. al., Working Group Report: Top Quark, in Proceedings, 2013 Community Summer Study on the Future of U.S. Particle Physics: Snowmass on the Mississippi (CSS2013): Minneapolis, MN, USA, July 29-August 6, 2013, 2013. 1311.2028.

[3] M. E. Luke and M. J. Savage, Flavor changing neutral currents in the Higgs sector and rare top decays, Phys. Lett. B307 (1993) 387-393, hep-ph/9303249.

[4] D. Atwood, L. Reina, and A. Soni, Phenomenology of two Higgs doublet models with flavor changing neutral currents, Phys. Rev. D55 (1997) 3156-3176, hep-ph/9609279.

[5] J. J. Liu, C. S. Li, L. L. Yang, and L. G. Jin, $t \rightarrow c V$ via SUSY FCNC couplings in the unconstrained MSSM, Phys. Lett. B599 (2004) 92-101, hep-ph/0406155]. 
[6] D. Delepine and S. Khalil, Top flavor violating decays in general supersymmetric models, Phys. Lett. B599 (2004) 62-74, [hep-ph/0406264].

[7] C. S. Li, R. J. Oakes, and J. M. Yang, Rare decay of the top quark in the minimal supersymmetric model, Phys. Rev. D49 (1994) 293-298. [Erratum: Phys. Rev.D56,3156(1997)].

[8] J. L. Lopez, D. V. Nanopoulos, and R. Rangarajan, New supersymmetric contributions to $t \rightarrow c$ V, Phys. Rev. D56 (1997) 3100-3106, hep-ph/9702350].

[9] J. M. Yang, B.-L. Young, and X. Zhang, Flavor changing top quark decays in r parity violating SUSY, Phys. Rev. D58 (1998) 055001, hep-ph/9705341.

[10] J. A. Aguilar-Saavedra, Top flavor-changing neutral interactions: Theoretical expectations and experimental detection, Acta Phys. Polon. B35 (2004) 2695-2710, hep-ph/0409342.

[11] J. A. Aguilar-Saavedra and G. C. Branco, Probing top flavor changing neutral scalar couplings at the CERN LHC, Phys. Lett. B495 (2000) 347-356, hep-ph/0004190.

[12] J. A. Aguilar-Saavedra and B. M. Nobre, Rare top decays $t \rightarrow c$ gamma, $t \rightarrow c g$ and CKM unitarity, Phys. Lett. B553 (2003) 251-260, hep-ph/0210360.

[13] S. Khatibi and M. Mohammadi Najafabadi, Constraints on top quark flavor changing neutral currents using diphoton events at the LHC, Nucl. Phys. $B 909$ (2016) 607-618, 1511.00220.

[14] H. Khanpour, S. Khatibi, M. Khatiri Yanehsari, and M. Mohammadi Najafabadi, Single top quark production as a probe of anomalous tq $\gamma$ and tqZ couplings at the FCC-ee, Phys. Lett. B775 (2017) 25-31, 1408.2090].

[15] S. Khatibi and M. Mohammadi Najafabadi, Probing the Anomalous FCNC Interactions in Top-Higgs Final State and Charge Ratio Approach, Phys. Rev. D89 (2014), no. 5054011 , 1402.3073 .

[16] J. Gao, C. S. Li, L. L. Yang, and H. Zhang, Search for anomalous top quark production at the early LHC, Phys. Rev. Lett. 107 (2011) 092002, [1104.4945].

[17] H. Khanpour, Probing top quark FCNC couplings in the triple-top signal at the high energy LHC and future circular collider, Nucl. Phys. B 958 (2020) 115141, [1909.03998].

[18] H. Hesari, H. Khanpour, and M. Mohammadi Najafabadi, Direct and Indirect Searches for Top-Higgs FCNC Couplings, Phys. Rev. D92 (2015), no. 11 113012, 1508.07579.

[19] H. Hesari, H. Khanpour, M. Khatiri Yanehsari, and M. Mohammadi Najafabadi, Probing the Top Quark Flavour-Changing Neutral Current at a Future Electron-Positron Collider, Adv. High Energy Phys. 2014 (2014) 476490, 1412.8572.

[20] S. M. Etesami and M. Mohammadi Najafabadi, Study of anomalous top quark FCNC interactions via $t W$-channel of single top, Phys. Rev. D81 (2010) 117502, 1006.1717.

[21] ATLAS Collaboration, G. Aad et. al., Search for flavour-changing neutral current top-quark decays to $q Z$ in $p p$ collision data collected with the ATLAS detector at $\sqrt{s}=8$ TeV, Eur. Phys. J. C76 (2016), no. 1 12, 1508.05796. 
[22] ATLAS Collaboration, G. Aad et. al., Search for single top-quark production via flavour-changing neutral currents at 8 TeV with the ATLAS detector, Eur. Phys. J. C76 (2016), no. 2 55, 1509.00294.

[23] ATLAS Collaboration, G. Aad et. al., A search for flavour changing neutral currents in top-quark decays in pp collision data collected with the ATLAS detector at $\sqrt{s}=7$ TeV, JHEP 09 (2012) 139, 1206.0257.

[24] CMS Collaboration, V. Khachatryan et. al., Search for Anomalous Single Top Quark Production in Association with a Photon in pp Collisions at $\sqrt{s}=8 \mathrm{TeV}$, JHEP 04 (2016) 035, 1511.03951.

[25] ATLAS Collaboration, G. Aad et. al., Search for flavour-changing neutral currents in processes with one top quark and a photon using $81 \mathrm{fb}^{-1}$ of pp collisions at $\sqrt{\mathrm{s}}=13 \mathrm{TeV}$ with the ATLAS experiment, Phys. Lett. B800 (2020) 135082, 1908.08461.

[26] ATLAS Collaboration, M. Aaboud et. al., Search for flavour-changing neutral current top-quark decays $t \rightarrow q Z$ in proton-proton collisions at $\sqrt{s}=13$ TeV with the ATLAS detector, JHEP 07 (2018) 176, 1803.09923.

[27] CMS Collaboration, S. Chatrchyan et. al., Search for Flavor Changing Neutral Currents in Top Quark Decays in pp Collisions at 7 TeV, Phys. Lett. B718 (2013) 1252-1272, 1208.0957 .

[28] CMS Collaboration, S. Chatrchyan et. al., Search for Flavor-Changing Neutral Currents in Top-Quark Decays $t \rightarrow Z q$ in pp Collisions at $\sqrt{s}=8$ TeV, Phys. Rev. Lett. 112 (2014), no. 17 171802, 1312.4194 .

[29] Do Collaboration, V. M. Abazov et. al., Search for Flavor Changing Neutral Currents in Decays of Top Quarks, Phys. Lett. B701 (2011) 313-320, [1103.4574.

[30] ZEUS Collaboration, H. Abramowicz et. al., Search for single-top production in ep collisions at HERA, Phys. Lett. B708 (2012) 27-36, [1111.3901].

[31] DELPHI Collaboration, J. Abdallah et. al., Search for single top production via FCNC at LEP at $\sqrt{s}=189-G e V$ to 208-GeV, Phys. Lett. B590 (2004) 21-34, hep-ex/0404014.

[32] CMS Collaboration, A. M. Sirunyan et. al., Search for associated production of a $Z$ boson with a single top quark and for $t Z$ flavour-changing interactions in pp collisions at $\sqrt{s}=8$ TeV, JHEP 07 (2017) 003, 1702.01404.

[33] M. Malekhosseini, M. Ghominejad, H. Khanpour, and M. Mohammadi Najafabadi, Constraining top quark flavor violation and dipole moments through three and four-top quark productions at the LHC, Phys. Rev. D 98 (2018), no. 9 095001, [1804.05598].

[34] CMS Collaboration Collaboration, Updates on Projections of Physics Reach with the Upgraded CMS Detector for High Luminosity LHC, .

[35] CMS Collaboration Collaboration, Projections for Top FCNC Searches in 3000/fb at the LHC, tech. rep., CERN, Geneva, 2013.

[36] FCC Collaboration, A. Abada et. al., FCC Physics Opportunities, Eur. Phys. J. C79 (2019), no. 6474. 
[37] FCC Collaboration, A. Abada et. al., FCC-ee: The Lepton Collider, Eur. Phys. J. ST 228 (2019), no. 2 261-623.

[38] M. Ahmad et. al., CEPC-SPPC Preliminary Conceptual Design Report. 1. Physics and Detector, .

[39] ILC Collaboration, H. Aihara et. al., The International Linear Collider. A Global Project, 1901.09829 .

[40] The International Linear Collider Technical Design Report - Volume 2: Physics, 1306.6352.

[41] H. Abramowicz et. al., The International Linear Collider Technical Design Report - Volume 4: Detectors, 1306.6329.

[42] CLIC Detector and Physics Study Collaboration, H. Abramowicz et. al., Physics at the CLIC e+e-Linear Collider - Input to the Snowmass process 2013, in Proceedings, 2013 Community Summer Study on the Future of U.S. Particle Physics: Snowmass on the Mississippi (CSS2013): Minneapolis, MN, USA, July 29-August 6, 2013, 2013. 1307.5288.

[43] I. Brivio and M. Trott, The Standard Model as an Effective Field Theory, Phys. Rept. 793 (2019) 1-98, 1706.08945.

[44] B. Grzadkowski, M. Iskrzynski, M. Misiak, and J. Rosiek, Dimension-Six Terms in the Standard Model Lagrangian, JHEP 10 (2010) 085, 1008.4884.

[45] J. A. Aguilar-Saavedra, A Minimal set of top anomalous couplings, Nucl. Phys. B812 (2009) 181-204, 0811.3842.

[46] C. Degrande, C. Duhr, B. Fuks, D. Grellscheid, O. Mattelaer, and T. Reiter, UFO - The Universal FeynRules Output, Comput. Phys. Commun. 183 (2012) 1201-1214, 1108.2040.

[47] J. Alwall, R. Frederix, S. Frixione, V. Hirschi, F. Maltoni, O. Mattelaer, H. S. Shao, T. Stelzer, P. Torrielli, and M. Zaro, The automated computation of tree-level and next-to-leading order differential cross sections, and their matching to parton shower simulations, JHEP 07 (2014) 079, 1405.0301.

[48] A. Amorim, J. Santiago, N. Castro, and R. Santos, http://feynrules.irmp.ucl.ac.be/wiki/GeneralFCNTop, .

[49] A. Alloul, N. D. Christensen, C. Degrande, C. Duhr, and B. Fuks, FeynRules 2.0 - A complete toolbox for tree-level phenomenology, Comput. Phys. Commun. 185 (2014) 2250-2300, 1310.1921.

[50] P. Skands, S. Carrazza, and J. Rojo, Tuning PYTHIA 8.1: the Monash 2013 Tune, Eur. Phys. J. C74 (2014), no. 8 3024, 1404.5630.

[51] DELPHES 3 Collaboration, J. de Favereau, C. Delaere, P. Demin, A. Giammanco, V. Lemaitre, A. Mertens, and M. Selvaggi, DELPHES 3, A modular framework for fast simulation of a generic collider experiment, JHEP 02 (2014) 057, 1307.6346.

[52] Particle Data Group Collaboration, M. Tanabashi et. al., Review of Particle Physics, Phys. Rev. D 98 (2018), no. 3030001. 
[53] M. Backovic, O. Gabizon, J. Juknevich, G. Perez, and Y. Soreq, Measuring boosted tops in semi-leptonic $t \bar{t}$ events for the standard model and beyond, JHEP 04 (2014) 176, 1311.2962 .

[54] G. Moortgat-Pick et. al., The Role of polarized positrons and electrons in revealing fundamental interactions at the linear collider, Phys. Rept. 460 (2008) 131-243, hep-ph/0507011. 